University of Wollongong

Research Online

Faculty of Engineering and Information

Faculty of Engineering and Information

Sciences - Papers: Part A

Sciences

2007

Polymer entanglement density and its influence on interfacial friction

Philip G. Whitten

University of Wollongong,whitten@uow.edu.au

Hugh R. Brown

University of Wollongong

Follow this and additional works at: https://ro.uow.edu.au/eispapers

Part of the Engineering Commons, and the Science and Technology Studies Commons

Research Online is the open access institutional repository for the University of Wollongong. For further information contact the UOW Library: research-pubs@uow.edu.au 


\title{
Polymer entanglement density and its influence on interfacial friction
}

\author{
Abstract \\ The entanglement density of amorphous glassy polymers is well known to control their fracture \\ mechanisms under tensile loading. There have been some reports indicating substantial deformation of a \\ glassy polymer's surface region when exposed to interfacial friction. It is shown conclusively here that \\ there is a direct correlation between the entanglement density of a glassy polymer and the deformation \\ mechanisms that facilitate sliding friction. This correlation was shown experimentally by studying the \\ topography of polymer surfaces following a single sliding pass by an inorganic glass sphere. Four \\ different polymers were studied, including polystyrene cross linked to different degrees. It is also shown \\ that permanent plastic deformation accompanies interfacial friction, and, furthermore, that the type of \\ deformation is a direct function of the respective polymer's entanglement density. In contrast, no \\ difference in the observed friction force could be attributed to the entanglement density of the respective \\ polymers. The findings can be explained by the state- and rate-dependent friction model. \\ Disciplines \\ Engineering | Science and Technology Studies

\section{Publication Details} \\ Whitten, P. G. \& Brown, H. R. (2007). Polymer entanglement density and its influence on interfacial friction. \\ Physical Review E (Statistical, Nonlinear, and Soft Matter Physics), 76 (2), 026101-1-026101-8.
}




\title{
Polymer entanglement density and its influence on interfacial friction
}

\author{
Philip G. Whitten and Hugh R. Brown \\ Materials Engineering, University of Wollongong, Wollongong, New South Wales 2522, Australia \\ (Received 18 July 2005; revised manuscript received 21 September 2006; published 1 August 2007)
}

\begin{abstract}
The entanglement density of amorphous glassy polymers is well known to control their fracture mechanisms under tensile loading. There have been some reports indicating substantial deformation of a glassy polymer's surface region when exposed to interfacial friction. It is shown conclusively here that there is a direct correlation between the entanglement density of a glassy polymer and the deformation mechanisms that facilitate sliding friction. This correlation was shown experimentally by studying the topography of polymer surfaces following a single sliding pass by an inorganic glass sphere. Four different polymers were studied, including polystyrene cross linked to different degrees. It is also shown that permanent plastic deformation accompanies interfacial friction, and, furthermore, that the type of deformation is a direct function of the respective polymer's entanglement density. In contrast, no difference in the observed friction force could be attributed to the entanglement density of the respective polymers. The findings can be explained by the state- and rate-dependent friction model.
\end{abstract}

DOI: 10.1103/PhysRevE.76.026101

PACS number(s): 81.40.Pq, 82.35.Lr, 68.35.Gy, 68.37.Ps

\section{INTRODUCTION}

Polymer films and their interactions with moving objects are critical to the production and lifetime of many components in the microelectronics industry. For example, the surfaces of thin polymer films are oriented by brushing with a velour cloth in the commercial production of liquid crystal displays, and the head of a hard drive may come into contact with a polymer-lubricant-coated disk in the operation of a hard drive. Indeed, the interaction between a polymer film and a moving object is of broad interest, as so many objects are now coated with a polymer film to provide a barrier coating to wear or the environment, to change the reflectance of light, or to change the surface properties and hence the adherence or wettability of substances to the surface.

With increasing emphasis in micro- and nanotechnology, and, specifically, microelectronics, components interacting with polymer films are becoming smaller within an operating environment that is becoming cleaner. One can predict that initial contacts between adjacent surfaces in such systems are not limited by the existence of wear debris, contaminant particles, or surface roughness that allow for the empirical friction law, but are governed by interfacial friction.

It is known from industry that plastic deformation of polymer coatings at some scales must accompany the friction processes. Orientation of polymer surfaces by brushing for liquid displays, or cross linking of polymer coatings to reduce wear for hard drives are two examples. It is also known that, for amorphous glassy polymers, the degree of chain entanglement, referred to as their entanglement density, controls the localization of strain, and hence their failure mechanism under applied load.

This work explores the relationship between polymer entanglement density, plastic deformation, and friction. We focus on a relatively simple sliding system, where a portion of a polymer film is subjected to a single sliding pass by an inorganic glass indenter. The principal goal is to explore the effects of polymer entanglement density on the permanent change in surface topography induced by a single sliding pass of an indenter.

\section{PRIOR WORK}

\section{A. Polymer entanglement and failure}

Polymer chains are linearly connected objects that entangle both inter- and intrachain when they are sufficiently long. The effect of molecular entanglements is made apparent by the occurrence of an intermediate rubbery plateau when shear modulus experiments are performed as a function of frequency at temperatures higher than the respective glass transition temperature [1]. The molecular weight between entanglements, $M_{e}$, can be calculated from the shear storage modulus $G$, at the point of inflection in the stress-strain curve using the theory of rubber elasticity:

$$
M_{e}=\frac{\rho R_{\text {gas }} T}{G^{\prime}},
$$

where $R_{\text {gas }}$ is the universal gas constant, $T$ is the absolute temperature, and $\rho$ is the mass density. The molecular weight between entanglements is unique for each polymer, and is controlled by a number of structural parameters, with chain stiffness being the most obvious. Such is their restriction to chain sliding that entanglements are often modeled as temporary physical cross links between polymer chains. Analogous to cross links, some authors prefer to refer to the density of entanglements known as the strand density $\nu_{E}$. A strand is defined as the length of polymer between two entanglements:

$$
\nu_{E}=\frac{\rho N_{A}}{M_{e}},
$$

where $N_{A}$ is Avogadro's number.

For glassy polymers, entanglements restrict the localization of strain by inhibiting the relative motion of polymer chains. Experimentally, it was shown for thin films of a series of homopolymers, copolymers, and polymer blends that crazing and shear yielding deformation varies systematically with $\nu_{E}$ [2]. Specifically, polymers with $\nu_{E}>8 \times 10^{25}$ chains $/ \mathrm{m}^{3}$ are unable to probe the fibrillation and void cre- 
ation processes essential for craze formation, and exhibit shear yielding under all multiaxial loading states [2]. Polymers with $\nu_{E}<4 \times 10^{25}$ chains $/ \mathrm{m}^{3}$ undergo craze deformation, leading to brittle failure in tension, but ductile deformation under compression or shear loading. Finally, polymers with $4 \times 10^{25}<\nu_{E}<8 \times 10^{25}$ chains $/ \mathrm{m}^{3}$ exhibit both crazing and shear yielding under tension. It is emphasized that polymers which exhibit crazing are brittle under tension because the local ductility of the polymer is so high that deformation occurs only over a very small region. Crazes typically form in primarily amorphous polymers in materials with molecular weights greater than a few times the entanglement molecular weight. The direction of craze growth is well defined, with the craze tip moving perpendicular to the maximum principal stress.

There are several ways one may alter the apparent entanglement density, including selecting different polymers; blending miscible polymers that have substantially different entanglement densities [3]; and cross-linking polymers [2]. Cross-linking polymers is the only technique that is able to shift the apparent entanglement density while maintaining a constant elastic modulus, yield strength, and surface energy.

For low cross-link densities, it is normally assumed that the cross links operate in a manner similar to the entanglements; hence the total strand density $\nu$ is [4]

$$
\nu=\nu_{x}+\nu_{E}
$$

where $\nu_{x}$ is the cross-link density.

\section{B. Interfacial sliding friction}

Friction is the resistance that bodies exert on each other when they are slid and/or rolled over each other. Amonton and Coulomb in the 17th and 18th centuries constructed the empirical friction law [5]

$$
F=\mu P,
$$

where $F$ is the friction force that opposes the motion of a body under normal load $P$, and $\mu$ is the friction coefficient, which is unique to the system being studied. In the early 20th century, several authors argued that surface roughness causes a large difference between the apparent contact area and the real contact area $A$ [6]. Consequently, it was proposed that asperities yield until the points of contact achieve the yield stress, and hence [7]

$$
A \alpha P, \quad F=\mu P=\tau A,
$$

where $\tau$ is the shear stress of the contact junction.

Homola et al. [8] reported that a number of different friction regimes exist. Normal friction exists when the contact between sliding surfaces is limited by contaminant particles, wear debris, or surface roughness, such that friction tends to follow the empirical friction law [9]. Interfacial friction occurs when the real area of contact is comparable to the apparent contact area, and the normal load is much less than that required for permanent deformation. Under interfacial friction conditions, the friction force is best described by

$$
F=S_{c} A,
$$

where $S_{c}$ is the critical shear stress [8]. $S_{c}$ is fundamentally different in origin from the shear stress $\tau$ proposed by Bowden and Tabor. The former is the stress required for sliding due to intermolecular forces, while the latter is the shear yield stress of the asperities that determines the contact area and the shear resistance of the contacts. For small applied loads, interfacial friction was shown to be much larger in magnitude than normal friction [8].

Recently, it was noticed for interfacial friction studies under applied load that the friction force was larger than that predicted by the critical shear stress and the contact area alone. A revised model incorporating Amontons' first law was proposed [10]:

$$
F=S_{c} A+\mu P .
$$

This revised model has the same form as the pressure dependence of friction reported by Bowers and Zisman [11], and then applied to polymers [12-16]. A major implication of the revised model is that the friction force cannot be described by the adhesive forces alone, but also has a loaddetermined term. When considering the pressure dependence of friction for polymers, the form of the equation was originally written as [16]

$$
F=\tau A=A\left(S_{c}+\alpha \sigma\right),
$$

where $\alpha$ is equivalent to $\mu$, and $\sigma$ is the average normal stress over the contact region; hence

$$
\sigma=\frac{P}{A} .
$$

$\alpha$ is analogous to the constant of proportionality in the MohrCoulomb yield criterion [17], which was shown to be appropriate for many materials, including the polymers tested in this paper, while $\mu$ originates from the friction properties of rough surfaces, where the contact area is proportional to the applied load.

\section{Brittle deformation due to interfacial friction}

Plastic or brittle deformation of a material's surface that was exposed to an interfacial friction process has not been widely reported. One reason is that the scale at which deformation would occur is smaller than that readily observed by optical microscopy.

Within the literature, there is strong evidence to believe that some permanent deformation must occur during the sliding process. Luengo et al. [18] reported the formation of 10 -nm-high ripples with a long wavelength on the surface of poly- $n$-butyl methacrylate when sliding it over a mica surface at $10^{\circ} \mathrm{C}$ below its glass transition temperature $\left(T_{g}\right)$. It was proposed that these ripples are short lived, as they could not be found after the two sliding surfaces were separated. In contrast, Cossy-Favre et al. [19] reported the orientation of a poly(biphenyltetracarboxylic dianhydride$p$-phenylenediamine) polyimide surface after being slid over by a glass lens. Polymer surfaces that have been buffed by a velour cloth in the production of liquid crystal displays have 
also been shown to be highly orientated in the surface region $[20,21]$.

If a polymer is undergoing deformation, then it is expected that the total strand density $\nu$ should determine the extent of local deformation. We report here permanent damage of a polymer surface due to interfacial friction, forming unique surface topographies. We show that $\nu$ determines the type of deformation that occurs. We also report that the interfacial friction force is not sensitive to variations in $\nu$ for a given polymer at low cross-link densities. These findings are consistent with a change in the differential of the wear rate versus $\nu$ noted for miscible blends of polystyrene (PS) and poly(2,6-dimethyl-1,4-phenylene oxide) (PPO) films [3].

Strain localization is the amount of deformation a local region may undergo before reaching a maximum and spreading to regions alongside. Under extreme strain localization, deformation becomes unstable, resulting in failure. Polymers that exhibit stable strain localization form a neck and draw under a tensile load; materials that undergo extreme strain localization exhibit fibrillation and craze growth prior to failure [22]. We show a direct link between strain localization and the plastic deformation occurring at a sliding interface.

\section{EXPERIMENT}

Polystyrenes with weight-averaged molecular weights $\left(\bar{M}_{w}\right)$ of 200000,51500 , and $3650 \mathrm{~g} \mathrm{~mol}^{-1}$ were obtained from Polysciences. Polymethylmethacrylate (PMMA), sold as ELVACITE 2021, was obtained from T. R. Chemicals, Australia. Poly(bisphenol $A$ carbonate) (PC) was obtained from Aldrich. Poly(2,6-dimethyl-1,4-phenylene oxide) was obtained from GE, USA. The respective polymers were typically spin coated onto polished silicon wafers (Silicon Inc., USA) from 2 wt \% solutions. Most of the films studied had a thickness of $60-100 \mathrm{~nm}$. The film thicknesses were measured using a Rudolph Research AutoEL-II elipsometer. When required, a range of polymer concentrations in solution were spin coated to achieve film thicknesses between $10 \mathrm{~nm}$ and $3 \mu \mathrm{m}$. The solvents used were toluene and/or tetrahydrofuran (HPLC grade, Aldrich). The polymer films were dried under vacuum at approximately $20^{\circ} \mathrm{C}$ above their respective $T_{g}$ 's. The roughness of the spincoated films was typically $<0.5 \mathrm{~nm}$ rms.

Some of the PS samples with a $\bar{M}_{w}$ of $200000 \mathrm{~g} \mathrm{~mol}^{-1}$ were cross linked using a Leica Stereoscan 440 scanning electron microscope using a method developed by Henkee and Kramer [2]. Briefly, PS samples were cross linked by imaging a region of the sample with a defocused beam using a beam intensity of $\sim 40 \mathrm{nA}$. The beam current was measured with a Keithley 486 picoammeter by focusing the beam on a $30 \mu \mathrm{m}$ aperture. This was done between each sample. After cross linking, the samples were left for 2 days for the long-lived free radicals, whose presence was suspected, to decay [23]. The cross-linked films were then washed with toluene and then dried at $120^{\circ} \mathrm{C}$ under vacuum. Film thickness measurements before cross-linking, and then after cross linking and washing with toluene, were used to calculate the soluble fraction, which, by applying the Charlsby-Pinner relationship [24], was used to calculate the

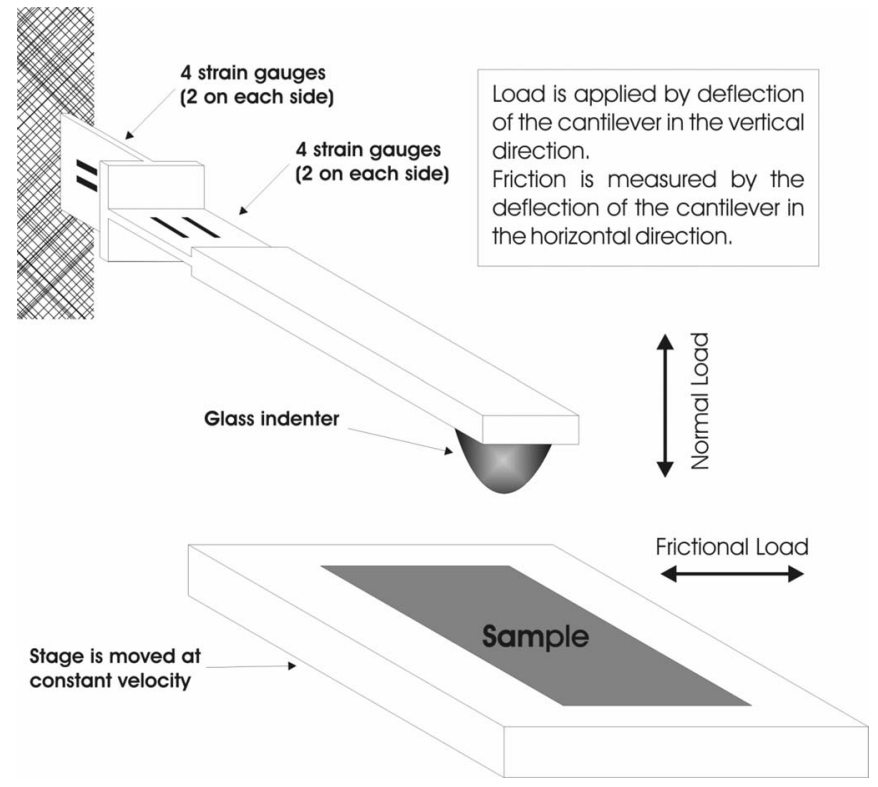

FIG. 1. Schematic of the friction apparatus.

gelation dose, and hence the cross-link density $\left(\nu_{x}\right)$ of the respective films.

All friction experiments were performed using a laboratory-built friction device (Fig. 1) at room temperature $\left(\sim 22{ }^{\circ} \mathrm{C}\right)$. Two types of glass indenters were used. Indenters of radii of curvature of $2-3 \mathrm{~mm}$ were made by melting the tip of an 8-mm-diameter soda glass rod, thereby forming a teardrop, of which the bottom was assumed to be spherical. The radius of curvature of the bottom was measured using an optical microscope. Other indenters with radii of curvature of $7.75 \mathrm{~mm}$ were lenses purchased from Edmund Optics (stock number D45-079). The indenters were mounted on the end of an aluminum double-cantilever beam. The roughness of the indenters obtained from Edmund Optics were between 0.7 and $3.5 \mathrm{~nm}$ rms, while that of the in-house-produced indenters was less than $0.5 \mathrm{~nm}$ rms. Normal load was applied by deflection of the beam. The friction and normal loads were measured separately by eight Entran semiconductor strain gauges (ESB-020-500) mounted on the cantilever in two standard Wheatstone bridge configurations. The samples were mounted on a Physik Instrumente (M-011.00) translation stage that was pushed by a Burleigh IW-710 series Inchworm motor over a distance of 5-10 mm. Except when explicitly stated, the presented results are for a sample velocity of $2 \mu \mathrm{m} \mathrm{s}^{-1}$. Multiple sliding passes of the same polymer surface were not studied.

The surfaces of the polymer samples were studied with a Digital Instruments Multimode scanning probe microscope (SPM) with a NanoScope IIIa controller in tapping mode.

\section{RESULTS}

\section{A. High-molecular-weight PS (200 $\left.000 \mathrm{~g} \mathrm{~mol}^{-1}\right)$, PMMA, PPO, and PC}

Two different modes of sliding were observed for each polymer system studied. In one mode, the friction force was 


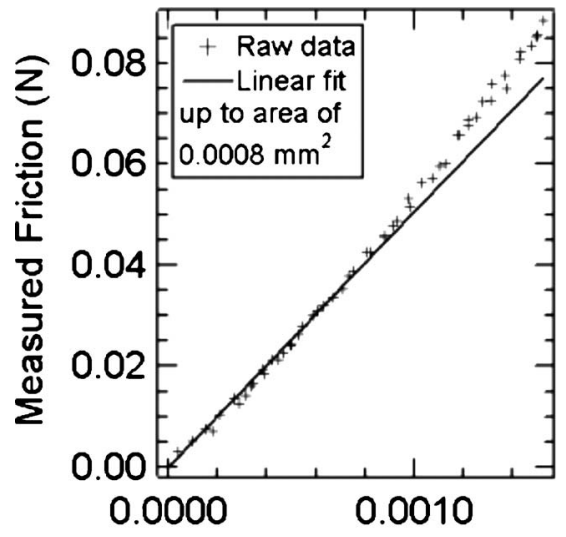

Hertzian Contact Area $\left(\mathrm{mm}^{2}\right)$

FIG. 2. Friction was not proportional to the contact area calculated by Hertzian contact mechanics. These results are from 2.47-mm-radius indenter sliding on PMMA.

relatively high and constant, and there was no evidence of surface damage by optical microscopy. For the other mode, the friction force was relatively low, and scratches were observed on the polymer surface by optical microscopy, together with delamination of pieces of the polymer coating. We assumed that the first mode was interfacial friction while the second mode was normal friction [8]. Typically, one could operate in the first mode for a period of time, before initiation of the second mode. The first mode was never observed to follow the second mode within a single slide. The initiation of the second mode is probably due to the occurrence of damage at the sliding interface. Indenters that were manufactured in house could be returned from the second mode to the first by rinsing the indenter with toluene, and then drying with nitrogen gas. The indenters purchased from Edmund Optics would produce the first mode only in the as-supplied condition, and exhibited only the second mode if they were washed or otherwise cleaned prior to experimentation. As we were concerned with sliding friction between two relatively smooth surfaces, we studied the first mode in great detail. We made no further study of the second mode.

PS, PPO, and PC all exhibited steady-state sliding after a sliding distance of $\sim 70 \mu \mathrm{m}$, regardless of the origin of the indenter. PMMA exhibited stick-slip sliding for the Edmund indenters, but steady-state sliding for the laboratory-made indenters (after a sliding distance of $\sim 70 \mu \mathrm{m}$. The friction forces presented here were obtained using the laboratorymade indenters, but were observed to be the same as the forces at the end of each slip region observed for the Edmund indenters. The magnitude of the stick-slip spikes for the Edmund indenters (and hence the force of maximum force of the stick) was consistent within a single slide, but varied between slides for the same experimental conditions.

For each polymer system studied, the friction force was proportional to the elastic contact area as calculated by Hertzian contact mechanics [25] for a single indenter at low loads (Fig. 2). However, indenters of different radii formed distinct groups when the measured friction was plotted against the Hertzian contact area. The friction force for different radii indenters was described well by considering the mean con-

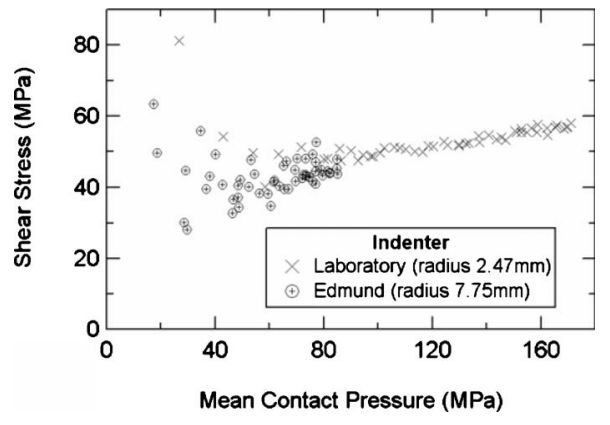

FIG. 3. The friction shear stress has a linear relationship to the mean contact pressure. These results are for indenters sliding on PMMA.

tact pressure that is normal to the polymer surface, between the indenter and the polymer (Fig. 3).

By using the least-squares method, a linear fit of the shear stress versus mean contact pressure was made for each of the polymer systems studied, with the results presented in Table I.

When studied by SPM, high-molecular-weight PS and PMMA each exhibited a surface topography within the rubbed region that was unique to the polymer system, and present for at least 3 months after the sliding experiments were performed. PC and PPO did not exhibit any characteristic surface features. Note that nothing was visible by optical microscopy for any of the samples determined to be operating in interfacial friction. For PS, the surface features consisted of elevated ridges that are oriented perpendicular to the sliding direction (Fig. 4). These ripples are not visible by optical microscope because of their very low aspect ratio. Typically, the ripples are $10-15 \mathrm{~nm}$ in height, and $300-500 \mu \mathrm{m}$ apart. No relationship between ripple spacing and indenter velocity $\left(1-500 \mu \mathrm{m} \mathrm{s}^{-1}\right)$ and/or applied load $(0.01-0.27 \mathrm{~N})$ could be determined for PS. Under low applied pressure the ripples were sharp (Fig. 5) relative to those formed under high applied pressure (Fig. 4). The form of the observed ripples was independent of film thickness over the complete range of film thickness studied $(15 \mathrm{~nm}-3 \mu \mathrm{m})$. The ripples were observed to form near the lead edge of contact (Fig. 6).

It is normally considered that, when brittle deformation and fracture accompany friction, then wear debris must be formed at the sliding interface, and so normal rather than

TABLE I. Fitting parameters for a linear fit of the shear stress versus the mean contact pressure. $S_{c}$ is the shear stress at zero applied pressure; $\alpha$ is the rate at which the shear stress increases with contact pressure as defined by Eq. (8). Mean contact pressures from 20 to $175 \mathrm{MPa}$ were studied at a sliding velocity of $2 \mu \mathrm{m} \mathrm{s}^{-1}$.

\begin{tabular}{ccc}
\hline \hline Polymer & $\begin{array}{c}S_{c} \\
(\mathrm{MPa})\end{array}$ & $\begin{array}{c}\alpha \\
\left(\mathrm{MPa} \mathrm{MPa}^{-1}\right)\end{array}$ \\
\hline PMMA & $38.6 \pm 1.4$ & $0.10 \pm 0.01$ \\
PS & $24.5 \pm 1.4$ & $0.13 \pm 0.01$ \\
PPO & $19.6 \pm 1.0$ & $0.05 \pm 0.01$ \\
PC & $16.6 \pm 5.2$ & $0.06 \pm 0.08$ \\
\hline \hline
\end{tabular}




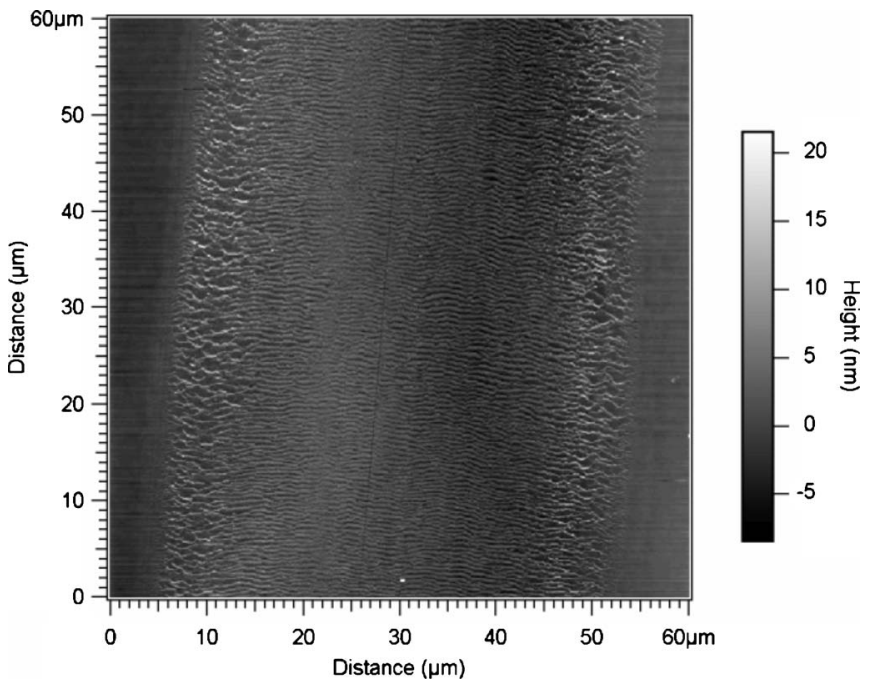

FIG. 4. A typical surface topography of an un-cross-linked PS surface after a single sliding pass. Sliding direction is bottom to top in all figures presented.

interfacial friction occurs [9]. Here, we report the formation of ripples that we assume to be wear debris, while maintaining a contact area sufficient to produce a high frictional force. Due to the high frictional force and the absence of scratches, we have used the term interfacial friction.

After a single sliding pass, the surface of PMMA samples exhibited a structure of raised isolated regions (Fig. 7). The dimensions and shapes of the isolated islands were variable even for the same apparent experimental conditions (indenter radii, applied load, sliding speed, and PMMA film thickness).

For both the PS and PMMA samples, the width of the sliding region as viewed by SPM showed an excellent correlation with the contact diameter predicted by Hertzian contact mechanics (Fig. 8). As the polymer film is so thin (between $15 \mathrm{~nm}$ and $3 \mu \mathrm{m}$ ) and hence confined, only the silicon substrate and the glass indenter were considered when calculating the contact diameter. More complicated contact me-

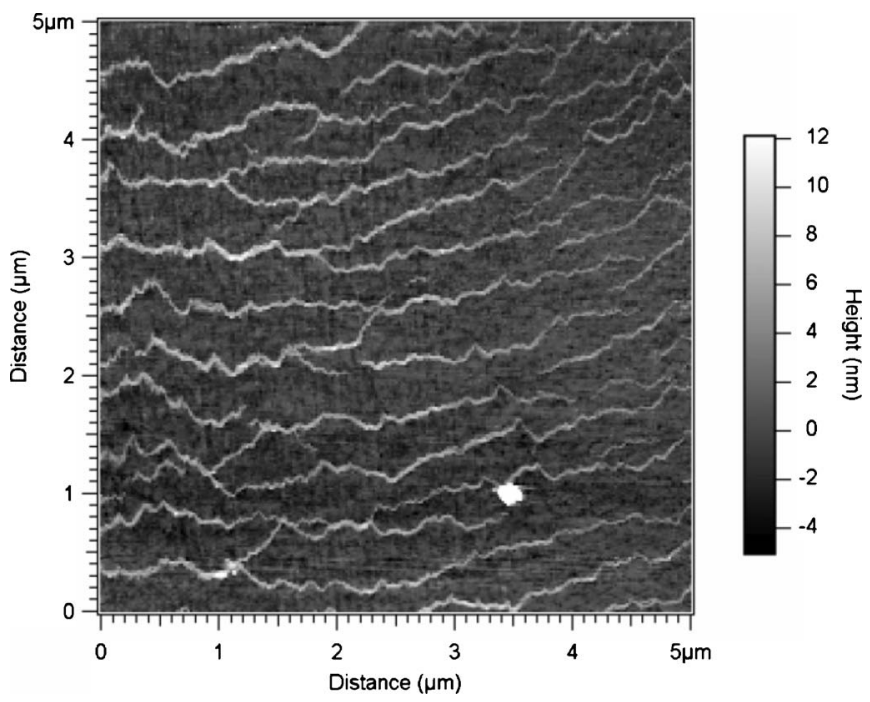

FIG. 5. Ridges on a PS under low contact pressure.

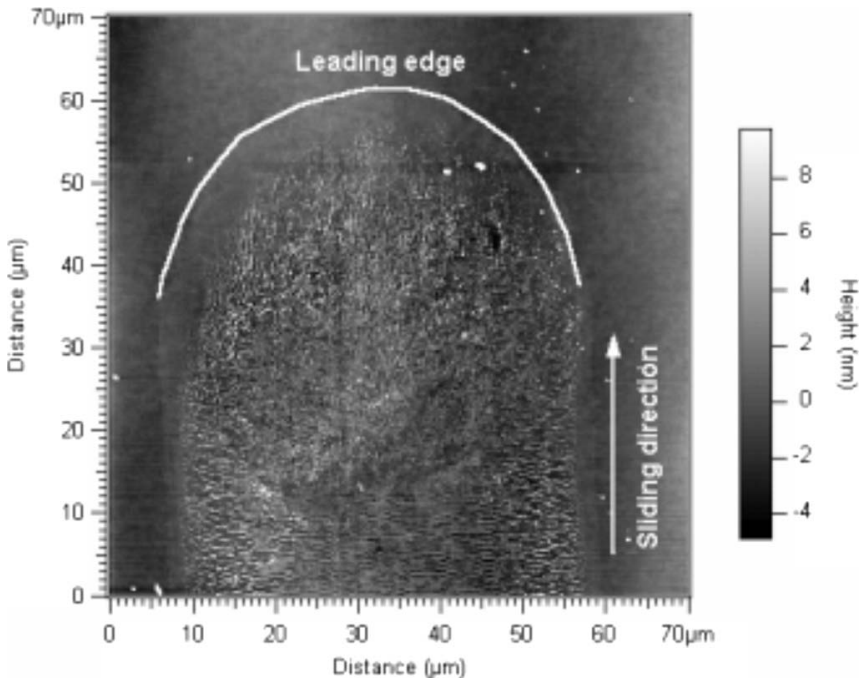

FIG. 6. The terminating end of a single slide. In this example the indenter was stopped and lifted from the sample. One can also see the results of the inevitable arcing movement of the double cantilever holding the indenter as it is raised.

chanics like the Johnson-Kendall-Roberts (JKR) [26] analysis were not considered, as the magnitude of the elastic moduli of the interacting surfaces, and the size of the contact area, ensure that the differences between JKR and Hertzian predictions would be small. Clearly, if one conducts experiments at loads much smaller than those presented here, then the contact area predicted from Hertzian contact mechanics would be too small.

\section{B. Cross-linked PS}

The PS samples were successfully cross linked, as confirmed by a $2.8 \times 1.7 \mathrm{~mm}^{2}$ rectangle of PS remaining on the $\mathrm{Si}$ substrate after washing with toluene. The observed friction force was independent of the cross-link density (Fig. 9). For cross-link densities less than or equal to $3.36 \nu_{E}$, ripples

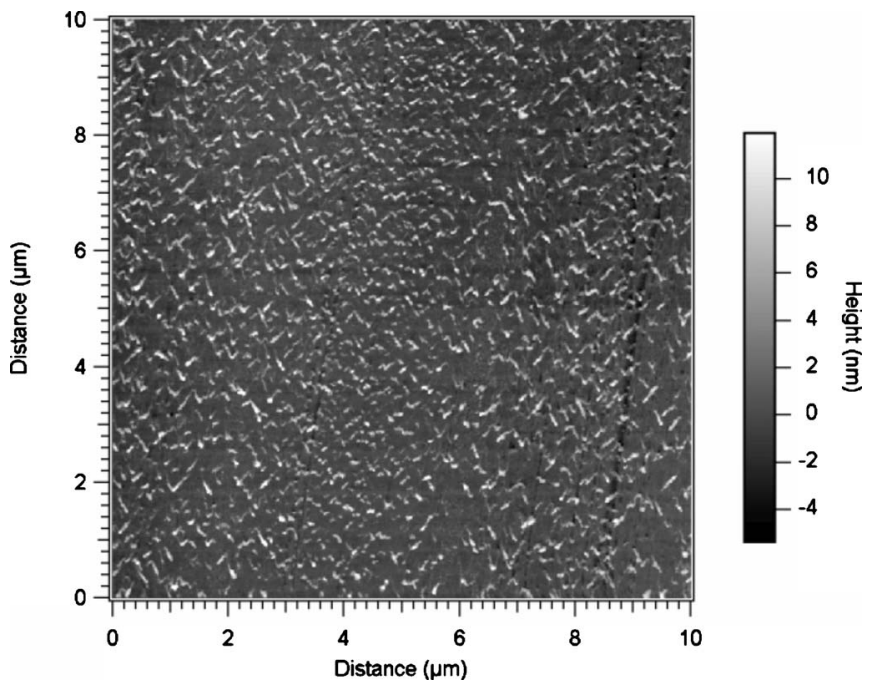

FIG. 7. Surface topography of a 15-nm-thick PMMA sample after a single sliding pass. 


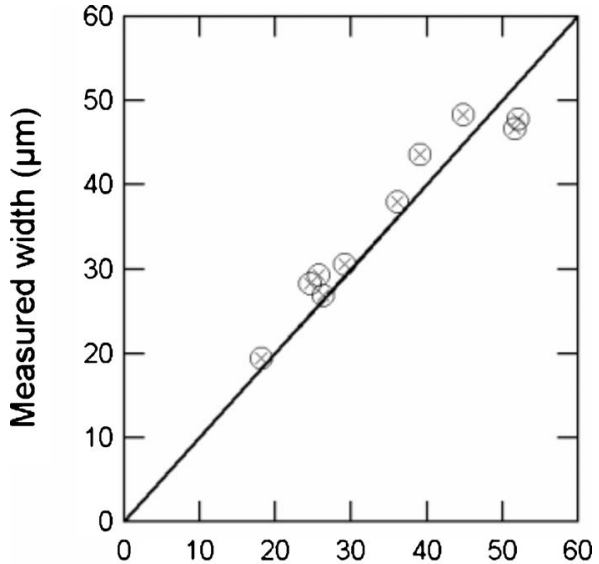

Hertzian contact diameter $(\mu \mathrm{m})$

FIG. 8. Width of contact sliding path measured by SPM compared to the contact diameter predicted by Hertzian contact mechanics. The solid line is a 1:1 relationship.

were observed on the surface by SPM. For cross-link densities greater than or equal to $4.54 \nu_{E}$, no characteristic surface features were visible by SPM.

\section{PS of different molecular weights}

When the rubbed region was studied by SPM, ripples as previously described were observed on the surfaces of PS with $\bar{M}_{w}$ of 200000 and $51500 \mathrm{~g} \mathrm{~mol}^{-1}$; however, the lowmolecular-weight sample $\left(\bar{M}_{w}=3650 \mathrm{~g} \mathrm{~mol}^{-1}\right)$ exhibited gross plastic deformation, but no ripple formation (Fig. 10). The friction force properties of the $\bar{M}_{w}=51500$ and $3650 \mathrm{~g} \mathrm{~mol}^{-1}$ samples were not studied.

\section{DISCUSSION}

The observed pressure-sensitive interfacial shear stress values (Table I) are in good agreement with those reported by Briscoe and Smith [15], as expected, because the experi-

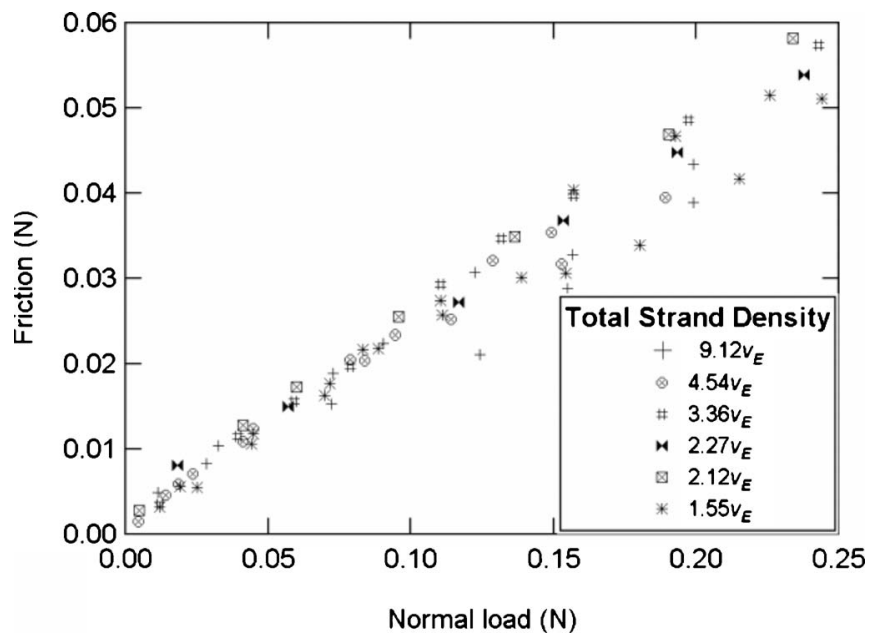

FIG. 9. Friction force versus applied load for different cross-link densities of PS at a sliding velocity of $2 \mu \mathrm{m} / \mathrm{s}$.

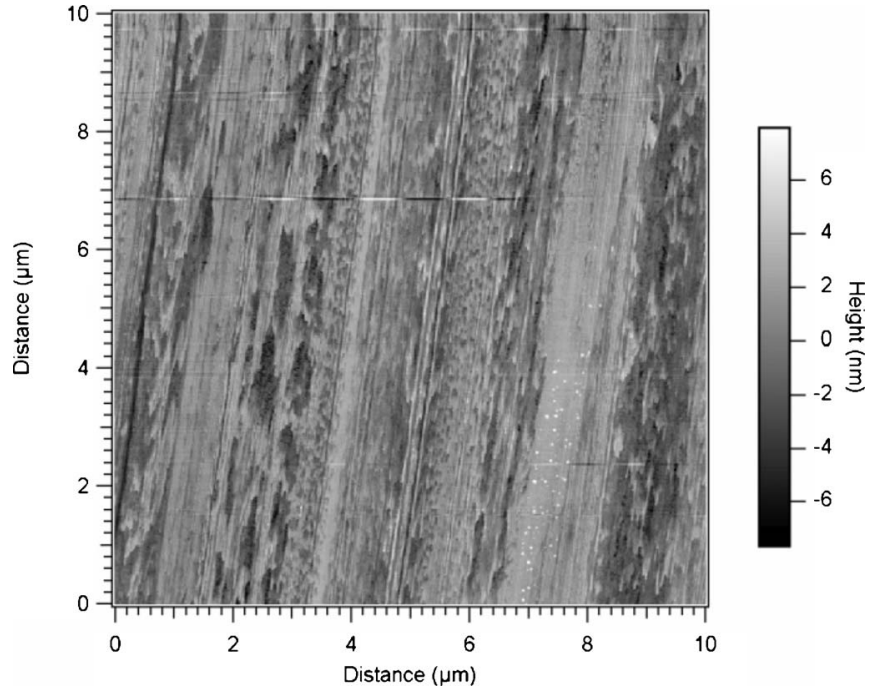

FIG. 10. High magnification of the plastic deformation on $\bar{M}_{w}$ $=3650 \mathrm{~g} \mathrm{~mol}^{-1}$ PS film.

mental design is similar. As originally indicated by Briscoe and Smith [15], a pressure-sensitive interfacial shear stress implies that polymer yield is a necessary precursor to sliding friction. Heuberger et al. [27] observed similar initial shear stress values $(\sim 10 \mathrm{MPa})$ for mica sliding on $\operatorname{poly}(n$-butyl methacrylate), even though their maximum applied normal stress was an order of magnitude lower than that reported here. Chen et al. [28] observed shear by stresses of about $\sim 1 \mathrm{MPa}$ for PS sliding on PS at normal stresses similar to those used Heuberger et al. [27]. Hence, it is assumed that the mode of friction observed here is similar to that observed by both Briscoe and Smith [15] and Heuberger et al. [27], but different from that observed by Chen et al. [28].

The interfacial friction force was observed to be independent of cross-link density. It has been suggested that the amount of friction energy consumed as a result of plastic work is less than $5 \%$ of the total frictional energy [29]. The only intrinsic polymer properties that could contribute to the observed friction force for nonpenetrating sliding surfaces are the surface energy, modulus, and yield stress. The surface energy determines the intermolecular forces, and hence adhesion at the sliding interface. The resistance to sheer is characterized by the yield stress and its pressure-sensitive coefficient $\alpha$. As demonstrated below, each of these should remain independent of the degree of cross linking for the range of cross-link densities studied. Studies have shown that cross linking does not alter the dynamic modulus of PS or isotactic PS below $T_{g}[30,31]$. As the microscopic mechanism of yielding is believed to involve chain motions over a length scale smaller than that of a strand, the cross-link densities used in this study would have little effect on the yield or flow stress [4]. Also, the proportion of segments involved in cross linking is very low, and hence no significant shift of the surface energy is expected. Hence, it was expected that the cross-link density of nonpenetrating polymer sliding surfaces should be independent of cross-link density.

In contrast to the observations presented here, Chen et al. $[28,32]$ showed for PS sliding on PS that cross linking an 


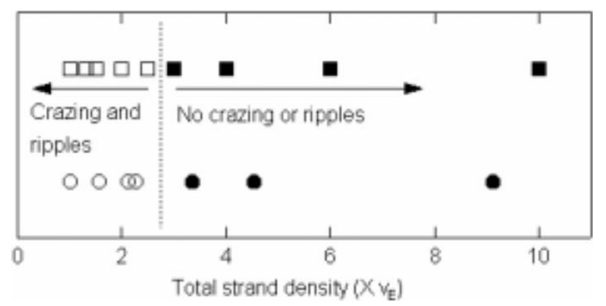

FIG. 11. The total strand density is consistent for ripple formation and craze formation for PS. (O) Ripples were observed by SPM. (@) No ripples were apparent by SPM. ( $\square$ ) Crazes form. (ם) No crazes form. Crazing results were taken from Henkee and Kramer [2].

un-cross-linked PS surface resulted in a substantial reduction of the frictional force. It was proposed that the observed reduction in friction force was due to penetrating polymer surfaces $[28,32]$. For the geometry employed in our work, the mechanism of polymer penetration is not possible, and hence a decrease in friction force due to an initial cross linking was not expected. It is pertinent to note that the mode of friction probed by Chen et al. $[28,32]$ is probably different from that probed in this study and by Heuberger et al. [27]. Evidence for the two different modes of interfacial friction is indicated by the fact that the interfacial shear stresses reported here and by Heuberger et al. [27] are an order of magnitude higher than those reported by Chen et al. [28,32]. For the friction mode presented here, the interfacial friction is sufficiently high that yielding of the PS surface is a necessary precursor to slip at the sliding interface. The same mode is essential to the orientation of polyimide surfaces in the production of liquid crystal displays [19-21]. In the friction mode presented by Chen et al. [28,32], the interfacial friction is sufficiently low that slip occurs at the sliding interface prior to the initiation of polymer yield. The strength of adhesion, and hence the degree of pinning at the sliding interface, determines which mode one operates in when the normal load is less than that required for plastic deformation alone [33].

The most startling observation was that, among four different polymers, those that have a strand density above a critical total strand density $\nu_{c}$ and are ductile under tensile deformation did not form characteristic surface features, while those that have a strand density below $\nu_{c}$ and are brittle under tensile load formed characteristic surface features. This trend was confirmed by studying different effective entanglement densities by cross-linking the PS and observing that the cross-link density where a transition from the occurrence of ripples to no ripples (hence from brittle to ductile failure) is the same as that for tensile loading (Fig. 11). Furthermore, the form of the surface features on PS was consistent with the ripples described by Homola et al. [8].

The strand density $\nu$ determines the degree of strain that can occur in a local region before strain is spread elsewhere. Hence strain localization must be associated with the cause of the ripples. Under tensile loading, polymers with a low $\nu$ undergo extreme local deformation, where through the process of craze formation the polymer will fail in an unstable and brittle fashion. Conversely, for polymers with a high $\nu$ the extent of local deformation is limited by the strand junctions, and the polymers will plastically deform in a stable manner.

It seems most likely that the characteristic surface features observed on PS and PMMA are the debris left behind after brittle deformation. These debris are fastened to the polymer surface by van der Waals forces. There must be intimate contact between the debris and the polymer surface due to their small size. They cannot be selectively removed mechanically, because one cannot get a handle on them. They cannot be removed chemically, because they are the same material as the substrate. Furthermore, we were unable to move them by using the atomic force microscope (AFM) in contact mode.

The appearance of the wear debris reported here are similar to those generated by scanning a standard AFM cantilever over a glassy polymer surface [34-36]. The wear debris resulting from scanning an AFM tip is believed to be the consequence of a peeling process as both the cutting and normal forces are relatively high [36]. In contrast, the wear debris observed here results from shear localizing within a segment of nanometer scale occurring under a relatively low normal force with a blunt indenter. Hence, scanning an AFM tip on PS with a total strand density greater than $\nu_{c}$ should generate wear debris while sliding with a blunt indenter should not.

For $\nu$, and hence strain localization, to determine when cohesive failure must occur a tensile stress must be present at the sliding interface. If one assumes that no slip occurs at the sliding interface, then a large tensile stress is expected at the trailing edge of the indenter, while a compressive stress is expected near the leading edge of the indenter [37,38]. A similar stress profile would develop if there is consistent sliding over the entire sliding interface. In both of these regimes one would expect to see ripple formation near the trailing edge only. However, the ripples were observed to begin forming near the leading edge (Fig. 6). Consequently, there must be local sticking regions and local slipping regions, with tensile stresses, and hence brittle failure occurs within each stick region within the predicted contact patch. During the microstick phase, the local regions in contact are deformed plastically, undergoing shear until the polymer begins to fail or the contact is broken, at which point a rapid slip motion occurs.

For contact between surfaces much rougher than those presented here, a multicontact interface results. It was shown that, for multicontact interfaces, sliding proceeds via the depinning of bistable "nanoblocks" of volume $\sim \mathrm{nm}^{3}$, which is known as the state- and rate-dependent friction model $[5,39,40]$. Recently, Bureau et al. [41] showed that, for a smooth PMMA indenter sliding over a glass surface, the interfacial shear stress increases logarithmically with sliding velocity. They proposed that the sliding interface is similar to that of a multicontact interface, where sliding proceeds through structural rearrangements of isolated nanoblocks at the PMMA surface $[33,41]$. The results presented here also indicate a sliding interface where there is structural rearrangement of clusters of volume $\sim \mathrm{nm}^{3}$.

This paper shows that the presence of wear debris at a sliding interface supports the notion of a sliding proceeding through the local shear of isolated nanoblocks within an in- 
terfacial regime, as predicted by the strain- and ratedependent friction model. The results of this paper imply that the properties of the polymer, as governed by its entanglement density, must contribute to the degree of shear within, and hence the size of the isolated clusters facilitating the sliding process. Consequently, the entanglement density of the polymer must contribute to the dynamics within a sliding interface.

\section{CONCLUSIONS}

We have shown that glassy polymers undergo plastic deformation when exposed to interfacial shear which is ex-

[1] R. S. Porter and J. F. Johnson, Chem. Rev. (Washington, D.C.) 66, 1 (1966).

[2] C. S. Henkee and E. J. Kramer, J. Polym. Sci., Polym. Phys. Ed. 22, 721 (1984).

[3] A. C.-M. Yang and W. W. Tsai, J. Polym. Sci., Part B: Polym. Phys. 35, 1295 (1997).

[4] L. L. Berger and E. J. Kramer, J. Mater. Sci. 23, 3536 (1988).

[5] B. N. J. Persson, Sliding Friction Physical Principles and Applications (Springer-Verlag, Berlin, 2000).

[6] J. F. Archard, Proc. R. Soc. London, Ser. A 243, 190 (1957).

[7] F. P. Bowden and D. Tabor, Proc. R. Soc. London, Ser. A 169, 391 (1939).

[8] A. M. Homola et al., Wear 136, 65 (1990).

[9] M. Godet, Wear 100, 437 (1984).

[10] J. N. Israelachvili et al., J. Mater. Res. 20, 1952 (2005).

[11] R. C. Bowers and W. A. Zisman, J. Appl. Phys. 39, 5385 (1968).

[12] R. C. Bowers, J. Appl. Phys. 42, 4961 (1971).

[13] J. K. A. Amuzu, B. J. Briscoe, and D. Tabor, ASLE Trans. 20, 354 (1977)

[14] L. C. Towle, J. Appl. Phys. 42, 2368 (1971).

[15] B. J. Briscoe and A. C. Smith, Polymer 22, 1587 (1981).

[16] B. J. Briscoe and A. P. Smith, J. Phys. D 15, 579 (1982).

[17] P. B. Bowden and J. A. Jukes, J. Mater. Sci. 3, 183 (1968).

[18] G. Luengo, M. Heuberger, and J. Israelachvili, J. Phys. Chem. B 104, 7944 (2000).

[19] A. Cossy-Favre et al., Macromolecules 31, 4957 (1998).

[20] M. G. Samant et al., Macromolecules 29, 8334 (1996).

[21] J. Stöhr et al., Macromolecules 31, 1942 (1998).

[22] P. Miller and E. J. Kramer, J. Mater. Sci. 25, 1751 (1990).

[23] L. A. Wall and D. W. Brown, J. Phys. Chem. 61, 129 (1957). plained by the strain- and rate-dependent friction model. Furthermore, the type of plastic deformation is determined by strain localization, and hence the entanglement density of the specific polymer. We have also shown that a polymer's entanglement density contributes to the mechanisms that occur at a sliding interface under conditions of interfacial friction. The entanglement density of a polymer determines some of the wear mechanisms that are able to take place at a sliding interface.

\section{ACKNOWLEDGMENTS}

This research was supported by the Australian Research Council.

[24] A. Charlesby and S. H. Pinner, Proc. R. Soc. London, Ser. A 249, 367 (1967).

[25] H. Hertz, J. Reine Angew. Math. 92, 156 (1881).

[26] K. L. Johnson, K. Kendall, and A. D. Roberts, Proc. R. Soc. London, Ser. A 324, 301 (1971).

[27] M. Heuberger, G. Luengo, and J. N. Israelachvili, J. Phys. Chem. B 103, 10127 (1999).

[28] N. Chen et al., Macromolecules 38, 3491 (2005).

[29] F. E. J. Kennedy, Wear 100, 453 (1984).

[30] M. Baccaredda, E. Butta, and V. Frosini, J. Appl. Polym. Sci. 10, 399 (1966).

[31] D. E. Kline, J. Appl. Polym. Sci. 5, 191 (1961).

[32] N. Maeda et al., Science 297, 379 (2002).

[33] L. Bureau, C. Caroli, and T. Baumberger, Phys. Rev. Lett. 97, 225501 (2006).

[34] G. F. Meyers, B. M. DeKoven, and J. T. Seitz, Langmuir 8, 2330 (1992).

[35] F. Dinelli, G. J. Leggett, and P. H. Shipway, Nanotechnology 16, 675 (2005).

[36] Z. Elkaakour, J. P. Aime, T. Bouhacina, C. Odin, and T. Masuda, Phys. Rev. Lett. 73, 3231 (1994).

[37] G. M. Hamilton, Proc. Inst. Mech. Eng., Part C: Mech. Eng. Sci. 197, 53 (1983).

[38] G. M. Hamilton and L. E. Goodman, J. Appl. Mech. 33, 371 (1966).

[39] T. Baumberger, P. Berthoud, and C. Caroli, Phys. Rev. B 60, 3928 (1999).

[40] B. N. J. Persson, Phys. Rev. B 63, 104101 (2000).

[41] L. Bureau, T. Baumberger, and C. Caroli, Eur. Phys. J. E 19, 163 (2006). 